\title{
RESEARCH
}

Open Access

\section{NRF2 is required for structural and metabolic maturation of human induced pluripotent stem cell-derived ardiomyocytes}

Xinyuan Zhang ${ }^{1,2}$, Liang $Y e^{1,2}$, Hao Xu $u^{2,3}$, Qin Zhou ${ }^{1,2}, \operatorname{Bin} \operatorname{Tan}^{1,2}$, Qin $Y i^{1,2}$, Liang Yan ${ }^{1,2}$, Min Xie ${ }^{1,2}$, Yin Zhang ${ }^{1,2}$, Jie $\operatorname{Tian}^{2,4}$ and Jing Zhu ${ }^{1,2^{*}}$

\begin{abstract}
Background: Human induced pluripotent stem cell-derived cardiomyocytes (hiPSC-CMs) hold great promise for regenerative medicine and in drugs screening. Despite displaying key cardiomyocyte phenotypic characteristics, they more closely resemble fetal/neonatal cardiomyocytes and are still immature; these cells mainly rely on glucose as a substrate for metabolic energy, while mature cardiomyocytes mainly employ oxidative phosphorylation of fatty acids. Studies showed that the alteration of metabolism pattern from glycolysis to oxidative phosphorylation improve the maturity of hiPSC-CMs. As a transcription factor, accumulating evidences showed the important role of NRF2 in the regulation of energy metabolism, which directly regulates the expression of mitochondrial respiratory complexes. Therefore, we hypothesized that NRF2 is involved in the maturation of hiPSC-CMs.

Methods: The morphological and functional changes related to mitochondria and cell maturation were analyzed by knock-down and activation of NRF2.

Results: The results showed that the inhibition of NRF2 led to the retardation of cell maturation. The activation of NRF2 leads to a more mature hiPSC-CMs phenotype, as indicated by the increase of cardiac maturation markers, sarcomere length, calcium transient dynamics, the number and fusion events of mitochondria, and mitochondrial respiration. Bioinformatics analysis showed that in addition to metabolism-related genes, NRF2 also activates the expression of myocardial ion channels.

(Continued on next page)
\end{abstract}

\footnotetext{
* Correspondence: jingzhu@cqmu.edu.cn

'Department of Pediatric Research Institute, Ministry of Education Key

Laboratory of Child Development and Disorders, National Clinical Research

Center for Child Health and Disorders (Chongqing), China International

Science and Technology Cooperation base of Child development and Critical

Disorders, Children's Hospital of Chongqing Medical University, Chongqing,

Box 136, No. 3 Zhongshan RD, Yuzhong district, Chongqing 400014, People's

Republic of China

${ }^{2}$ Chongqing Key Laboratory of Pediatrics, Chongqing, People's Republic of

China

Full list of author information is available at the end of the article
}

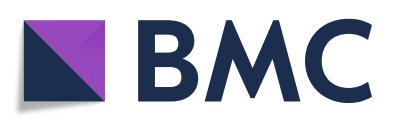

(c) The Author(s). 2021 Open Access This article is licensed under a Creative Commons Attribution 4.0 International License, which permits use, sharing, adaptation, distribution and reproduction in any medium or format, as long as you give appropriate credit to the original author(s) and the source, provide a link to the Creative Commons licence, and indicate if changes were made. The images or other third party material in this article are included in the article's Creative Commons licence, unless indicated otherwise in a credit line to the material. If material is not included in the article's Creative Commons licence and your intended use is not permitted by statutory regulation or exceeds the permitted use, you will need to obtain permission directly from the copyright holder. To view a copy of this licence, visit http://creativecommons.org/licenses/by/4.0/. The Creative Commons Public Domain Dedication waiver (http://creativecommons.org/publicdomain/zero/1.0/) applies to the data made available in this article, unless otherwise stated in a credit line to the data. 
(Continued from previous page)

Conclusions: These findings indicated that NRF2 plays an important role in the maturation of hiPSC-CMs. The present work provides greater insights into the molecular regulation of hiPSC-CMs metabolism and theoretical basis in drug screening, disease modeling, and alternative treatment.

Keywords: Nuclear factor erythroid 2 p45-related factor 2 (NRF2), Human induced pluripotent stem cell-derived cardiomyocytes (hiPSC-CMs), Metabolism

\section{Background}

Because of the irreparability of cardiac tissue and the scarcity of organs for donation, human induced pluripotent stem cell-derived cardiomyocytes (hiPSC-CMs) hold great potential for the treatment of myocardial injury [1]. However, multiple studies indicated that although hiPSC-CMs demonstrate obvious spontaneous beating; their metabolism, morphology, and structural functions are similar to those of embryonic immature cardiomyocytes $[2,3]$. The differences between mature and immature cardiomyocytes are manifested in many cellular aspects [4]. Compared with the morphology of embryonic cardiomyocytes, mature ones are larger, with significantly increased number of nucleus and more uniform cell pulsation. In the cellular ultrastructure, the number, volume, fusion, and cristae density of mitochondria are increased in mature hiPSC-CMs [5]. The mitochondria of embryonic cardiomyocytes are underdeveloped; therefore, these cells mainly rely on glucose as a substrate for metabolic energy, while mature cardiomyocytes mainly employ oxidative phosphorylation of fatty acids to fulfill the rising demand for energy [6]. Studies showed that the alteration of metabolism pattern from glycolysis to oxidative phosphorylation improves the maturity of hiPSC-CMs $[7,8]$.

The cap'n'collar basic leucine zipper (CNC-bZip) transcription factor, nuclear factor erythroid 2 p 45 -related factor 2 (NRF2), is the major regulator of cellular redox homeostasis. NRF2-targeted genes generate a large network of antioxidant enzymes, proteins, and transcriptional factors involved in energy metabolism, mitochondrial function, drug metabolism, proteasome degradation, and DNA repair [9]. In recent years, accumulating evidences showed the important role of NRF2 in the regulation of energy metabolism. A study by Ramachandra et al. showed that the expression of NRF2 significantly elevated in hiPSC-CMs which matured by fatty acids (FA) [10]. In addition, in the mitochondria of neurons and mouse embryonic fibroblasts, loss of NRF2 leads to mitochondrial respiration damage and decreased ATP levels and mitochondrial membrane potential, whereas genetic activation of NRF2 increases the rate of respiration, the efficiency of oxidative phosphorylation, and ATP levels [11]. It has been determined in different experimental systems that NRF2 directly regulates the expression of mitochondrial respiratory complexes such as ATP synthase subunit $\alpha$, NDUFA4, and cytochrome $\mathrm{C}$ oxidase subunits COX2 and COX4B $[12,13]$. In addition to mitochondrial respiration, NRF2 also promotes fatty acid $\beta$-oxidation metabolism [14]. Moreover, some studies show that NRF2 protects myocardium from damage due to its effects on antioxidative stress and promotion of mitochondrial aerobic respiration [15]. Therefore, we hypothesized that NRF2 is involved in the maturation of hiPSC-CMs. In this study, the morphological and functional changes related to mitochondria and cell maturation were analyzed by knockdown and activation of NRF2. The results showed that the inhibition of NRF2 led to the retardation of cell maturation, while the activation of NRF2 significantly promoted the cell maturation, which indicated the essential function of NRF2 in the maturation of hiPSC-CMs.

\section{Materials and methods}

Culture and cardiac differentiation of human induced pluripotent stem cell

Undifferentiated human epithelial cells of the urinederived iPS cells (hiPSCs), derived from CELLAPY Biological Technology (China), were maintained on Matrigel (BD Biosciences)-coated 6-well cell culture plates with PGM1 (CELLAPY Biological Technology). For cardiac cell differentiation, hiPSCs at $\sim 80 \%$ confluence was dissociated using EDTA (CELLAPY Biological Technology) and plated onto the Matrigel-coated 6-well plates (Corning) at a density of $4 \times 10^{5}$ cells/well. When the cells reached 90-98\% confluency, typically 4 days after passage, the medium was changed to RPMI plus B27 without insulin (Life Technologies) supplemented with $6 \mu$ M GSK3- $\beta$ inhibitor CHIR99021 (CHIR) (Selleck). Forty-eight hours later, the medium was completely replaced with RPMI/B27 medium without insulin and incubated for $24 \mathrm{~h}$. Then, the RPMI/B27 medium without insulin was exchanged with $5 \mu \mathrm{M}$ Wnt inhibitor IWP2 (Selleck). On day 5, the medium was refreshed with RPMI/B27 medium without insulin and incubated until day 7. The medium was then exchanged every 3 days with RPMI/B27 medium (Life Technologies). During day 13 to 16 , metabolic purification of the cardiomyocytes was performed by replacing the medium with RPMI medium without glucose and supplemented with B27 and $4 \mathrm{mM}$ sodium L-lactate (Sigma) [16]. On day 18 
of cardiac cell differentiation, the cells were dissociated with TrypLE Express Enzyme (Gibco) and seeded into Matrigel-coated 12-well plates (Corning) in RPMI/B27 medium. Then, cell cultures containing $>90 \%$ cardiac troponin T-positive CMs (confirmed by flow cytometry) were obtained.

\section{siRNA transfection}

The siRNAs used in this study were stealth-siRNA human NFE2L2 (NRF2, Thermo Fisher Scientific, MA), human KEAP1 siRNA (RIB BIO, MO), and negative control siRNA. The siRNAs were transfected into CMs with Lipofectamine RNAiMAX Reagent (Thermo Fisher Scientific, MA) at a concentration of $70 \mathrm{nM}$ in each well of a 12-well plate according to the manufacturer's recommendations. siRNA transfection was performed on day 24 of cardiac cell differentiation, and the cells were analyzed 3 days later.

\section{Immunostaining and confocal microscopic imaging Immunofluorescence staining}

Following 3 washes with PBS, cells (plated on a $1 \times 1 \mathrm{~cm}$ glass slide) were fixed for $20 \mathrm{~min}$ with $4 \%$ paraformaldehyde and washed again with PBS. The samples were then incubated for $10 \mathrm{~min}$ at room temperature with $0.5 \%$ Triton X-100 in PBS and blocked with 5\% bovine serum albumin (BSA) in PBS for $30 \mathrm{~min}$ at room temperature. Primary antibodies were diluted in 5\% BSA in PBS and incubated overnight at $4{ }^{\circ} \mathrm{C}$. Following 3 washes with $0.5 \%$ Triton X-100 in PBS, the samples were incubated with secondary antibodies diluted in 5\% BSA for $60 \mathrm{~min}$ at $37^{\circ} \mathrm{C}$. The samples were then washed, incubated with Hoechst 33342 (Beyotime, China) in PBS for $30 \mathrm{~min}$ at $37^{\circ} \mathrm{C}$ and washed again before imaging. Immunofluorescence staining was imaged using an A1R confocal microscope (Nikon, Japan). The following antibodies were used in this study: anti-SOX2, anti-Nanog (Proteintech, China), anti-cTnT, anti-CX43 (Abcam, China), and anti- $\alpha$-actinin (Proteintech, China). The secondary antibodies were goat anti-rabbit $\mathrm{Cy} 3$, rabbit anti-mouse Cy3 (CWBIO, Beijing, China), and goat anti-rabbit 488 (ZSGB-Bio, Beijing, China). Sarcomere lengths and nuclear numbers were measured by ImageJ software. $n>10$ cells per condition, three biological replicates. The lengths of ten sarcomeres from each cell were measured and averaged for each condition.

\section{Mitochondrial staining}

hiPSC-CMs were stained with either JC-1 dye (Beyotime, China) or prewarmed MitoTracker Red $(0.2 \mu \mathrm{M}$; Beyotime, China) for $25 \mathrm{~min}$ at $37^{\circ} \mathrm{C}$ according to the manufacturers' instructions. For mitochondrial staining, the nucleus was stained first. Ten microliters Hoechst dye was added to $1 \mathrm{ml}$ of medium, incubated for $16 \mathrm{~min}$ at $37^{\circ} \mathrm{C}$, and washed three times with PBS. Then, the mitochondria were stained. The staining and fluorescence intensity of the cells was evaluated using an A1R confocal microscope (Nikon, Japan). The exact amount of ATP is determined by an ATP determination kit (Beyotime, China) according to the manufacturer's instructions.

\section{Calcium imaging}

Following 3 washes with D-PBS, $0.3 \mathrm{mM}$ Fluo-4 AM (Beyotime) was added to the cells for $40 \mathrm{~min}$ at $37^{\circ} \mathrm{C}$, and then, the cells were washed with D-PBS for 3 times. The cells were incubated at $37{ }^{\circ} \mathrm{C}$ for $20 \mathrm{~min}$ before observation with A1R confocal microscope (Nikon, Japan). Videos were captured in continuous shooting mode using 488 channels.

\section{Ethynyl-20-Deoxyuridine (EdU) cell proliferation assay}

Cell proliferation was assessed with a BeyoClick ${ }^{\mathrm{Tw}}$ EdU cell proliferation kit with Alexa Fluor 488 (Beyotime, China). After $6.67 \mu \mathrm{M}$ EdU was added to cells, the cells were cultured for $15 \mathrm{~h}$ at $37^{\circ} \mathrm{C}$ to incorporate the reagent. After the cTnT of hiPSC-CMs were labeled using immunofluorescence, the cells then were incubated in Click Additive Solution and protected from light, washed three times with 3\% BSA in PBS, and stained with Hoechst 33342. Fluorescence images were obtained using an A1R confocal microscope (Nikon, Japan). The ratio of proliferating cells was determined by ImageJ software.

\section{Flow cytometry}

Following 3 washes with D-PBS, cells were fixed for 20 min with $4 \%$ (vol/vol) paraformaldehyde. The samples were then incubated for $10 \mathrm{~min}$ at room temperature with $0.5 \%$ Triton $\mathrm{X}-100$ in PBS, blocked for $30 \mathrm{~min}$ at room temperature with $5 \%$ bovine serum albumin in PBS, and labeled with rabbit anti-cTnT/AF488 (Bioss antibodies) in PBS. The cells were analyzed with a BD FACSCanto analyzer (BD Biosciences). Data analysis was performed using FlowJo software.

\section{Analysis of mtDNA copy number}

To evaluate mtDNA copy number, genomic and mitochondrial DNA was extracted with genomic DNA extraction kit (BioFlux). The extracted DNA was used as template for quantitative PCR (QuantStudio 3; Thermo Fischer Scientific) with a TB Green Premix Ex Taq kit (Roche, Basel, Switzerland) according to the manufacturer's instructions. The mitochondrial DNA (mt-ND1) to nuclear DNA ( $\beta$-globin) ratio was calculated as the mtDNA copy number. Error bars indicate the standard deviation of triplicate measurements of three biological samples. 


\section{Transmission electron microscopy (TEM)}

Cells with a density greater than $1 \times 10^{6}$ were collected in a $1.5-\mathrm{ml}$ centrifuge tube, fixed with $2.5 \%$ glutaraldehyde for $24 \mathrm{~h}$, and observed under a transmission electron microscope (TEM, Hitachi-7500, Japan) after rinsing, dehydration, soaking, and embedding.

\section{Mitochondrial function assay \\ Mitochondrial respiration}

The mitochondrial oxygen consumption rate (OCR) was assessed by an Agilent Seahorse XF Cell Mito Stress Test Kit and a 24-well XF cell culture microplate (Agilent Technologies, CA, USA) with nearly $1 \times 10^{5}$ cells added to each well. Before the assay, the cells were maintained on Seahorse XF base medium containing 1 M glucose, $100 \mathrm{mM}$ pyruvate, and $200 \mathrm{mM} \mathrm{L}$-glutamine. During OCR assessment, oligomycin $(1.5 \mu \mathrm{M})$, FCCP $(2 \mu \mathrm{M})$, and antimycin $\mathrm{A} /$ rotenone $(0.5 \mu \mathrm{M})$ were added to the system. Basal respiration, proton leakage, maximal respiration, ATP product, and spare respiratory capacity were measured in a XF24 analyzer.

\section{FAO assay}

Fatty acid oxidation (FAO) was assessed by an Agilent Seahorse XF Substrate Oxidation Stress Test Kit, same with mitochondrial stress test. Except for OCR assessment, there are four reagents Etomoxir (Eto, $4 \mu \mathrm{M})$, oligomycin $(1.5 \mu \mathrm{M})$, FCCP $(2 \mu \mathrm{M})$, and antimycin $\mathrm{A} /$ rotenone $(0.5 \mu \mathrm{M})$ were added to the system, other operation of the experiment is similar to Mito Stress Test. Basal respiration, proton leakage, maximal respiration, ATP product, and spare respiratory capacity were measured in a XF24 analyzer.

\section{Glycolysis analysis}

The glucose and lactic acid content detection kits (Solarbio, China) were used to detected glucose content and lactic acid content of cells, respectively. According to the original glucose and lactic acid content in the medium, the glucose consumption and lactic acid production are calculated, and the results are calibrated with protein content.

\section{Western blot analysis}

Western blot experiments were performed to determine the protein expression in cell lysates. The protein concentration was quantified with BCA protein quantitation assay (KeyGen Biotech, China). Equal protein amounts $(30 \mu \mathrm{g})$ were loaded onto SDS-PAGE gels and transferred to PVDF membranes (Millipore Sigma, China). Phosphorylated protein or common protein was blocked for $2 \mathrm{~h}$ at room temperature in 5\% BSA or 5\% skim milk in TBS with $0.05 \%$ Tween-20. Protein bands were probed overnight with the suitable primary antibody at
$4{ }^{\circ} \mathrm{C}$. Proteins were visualized using HRP-conjugated secondary antibody and a chemiluminescent detection kit (Millipore, USA). The amount of target protein was calculated by gray scanning.

\section{RNA extraction and quantitative PCR}

Total RNA was extracted with RNAiso Reagent (TaKaRa, Japan), and the RNA solution was obtained after chloroform extraction, isopropanol precipitation, ethanol washing, and RNase-free water dissolution. cDNA synthesis was completed with PrimeScript ${ }^{\mathrm{mi}}$ RT reagent kit with gDNA Eraser (TaKaRa). mRNA expression was measured by RT-PCR using a TB Green Premix Ex Taq kit (Roche, Basel, Switzerland) on a QuantStudio 3 Real-Time PCR System (Thermo Fischer Scientific). The reaction conditions were as follows: initial denaturation at $95^{\circ} \mathrm{C}$ for $10 \mathrm{~min} ; 39$ cycles of denaturation at $95^{\circ} \mathrm{C}$ for $5 \mathrm{~s}$, annealing, and extension at $60^{\circ} \mathrm{C}$ for $30 \mathrm{~s}$; and a melt curve reaction cycle. The fold change in expression was calculated with $2^{-\Delta \Delta C T}$ method with GAPDH RNA as the endogenous control.

\section{Statistical analysis}

All experiments showed in this research have been repeated more than three times. The data were analyzed by unpaired $t$-tests or Mann-Whitney $U$ test after a demonstration of homogeneity of variance with the $F$ test. Differences at a $P$ value of $<0.05$ were considered significant. Statistical analysis was performed by GraphPad Prism, version 7.

\section{Results}

\section{Characteristics of the hiPSCs and hiPSC-CMs}

The process of hiPSCs differentiation into cardiomyocytes (hiPSC-CMs) is shown in Fig. S1A. By regulating Wnt signal at different time points during differentiation, cardiomyocytes capable of spontaneous beating were obtained on the 13th day of induction (Movie S1) [17, 18]. On day 20 of cardiac cell differentiation, the positive rate of cTnT in the hiPSC-CMs was more than $98 \%$, as determined by flow cytometry (Fig. 1d). Pluripotency of hiPSC was further confirmed by immunocytochemistry (ICC) assay (Fig. S1B) and Q-PCR measurements (Fig. S1C, D). As shown in Supplement Fig. 1C and D, during the differentiation process, the mRNA expression levels of pluripotent markers were significantly decreased. In addition, the expression of cardiac marker in differentiated hiPSC-CMs was observed with immunofluorescence (Fig. 1a), and the expression levels was gradually increased with differentiation (Fig. 1b). Transmission electron microscopy showed that the differentiated hiPSC-CMs displayed a myocardialspecific sarcomere structure (Fig. 1c). These results demonstrated that the successfully differentiated cardiomyocytes were obtained. 

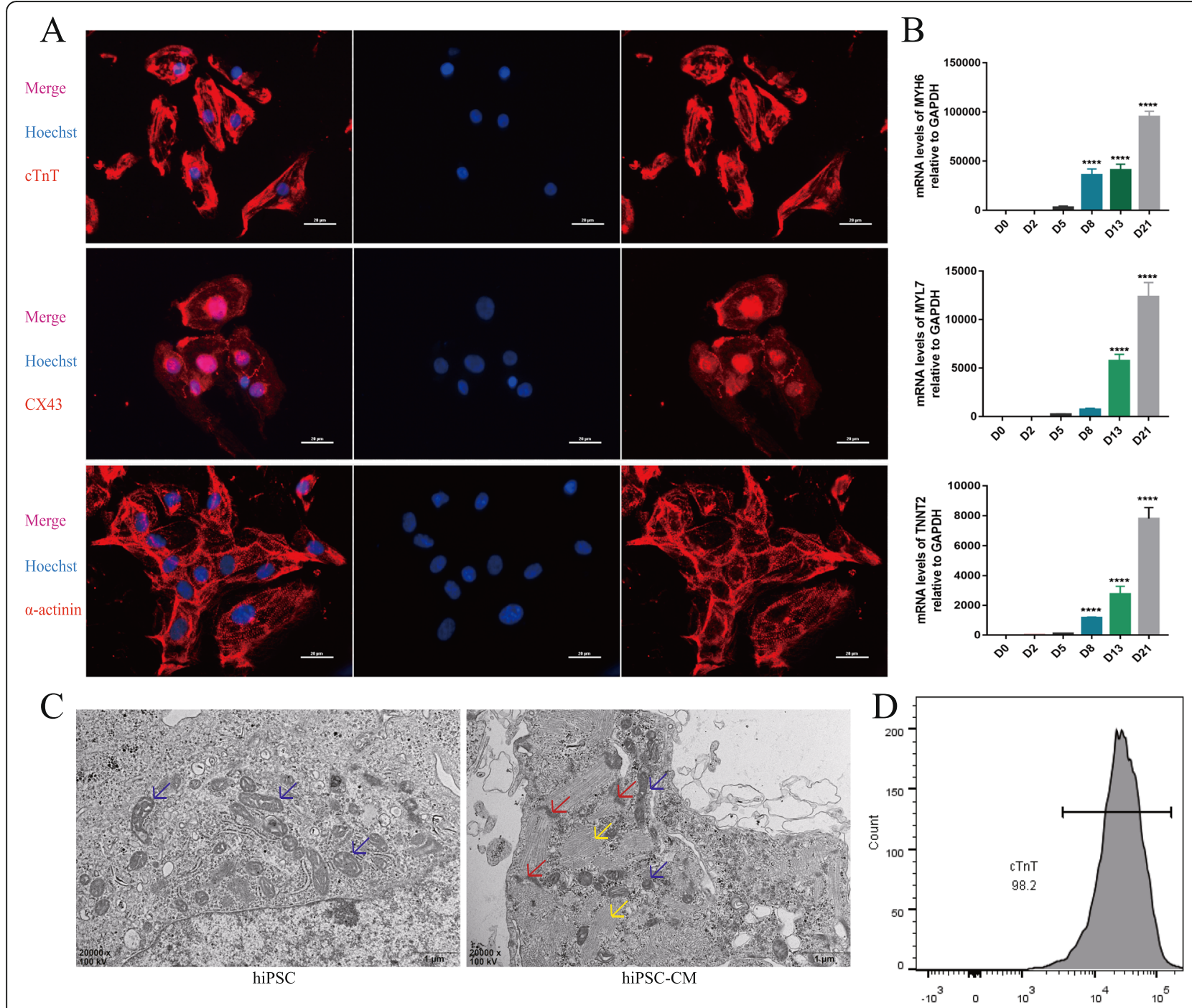

Fig. 1 Characterization of hiPSC-CMs. a Immunostaining of hiPSC-CMs for CTnT, CX43, and a-actinin. b Q-PCR of cardiac marker genes MYH6, MYL7, and TNNT2 during differentiation. The abscissa represents the number of days of differentiation. $n=3$; the means \pm SEM are shown. ${ }^{* * * *} P<0.0001$. $\mathbf{c}$ TEM images of hiPSC and hiPSC-CMs, myofibrils (yellow arrow), Z-disks (red arrow), and mitochondria (blue arrow). $\mathbf{d}$ Flow cytometry analysis of the hiPSC-CMs by cTnT measurements demonstrated cardiac cell purity greater than $98 \%$

\section{Inhibition of NRF2 suppresses hiPSC-CMs maturation}

To test the role of NRF2 in hiPSC-CMs, the NRF2 gene was knocked down by siRNA. NRF2 expression was significantly down-regulated in the NRF2-inhibited hiPSCCMs (siNRF2) compared with that in the control hiPSCCMs (siNC) by Q-PCR and Western blotting (Fig. 2a, b). Firstly, the effects of NRF2 knock-down on the morphology and function of hiPSC-CMs were investigated. Encouragingly, the inhibition of NRF2 caused a significant decrease of cardiac maturation markers, including potassium (KCND3 and KCNJ2) and calcium (CACNA1C, an L-type $\mathrm{Ca}$ channel protein, correlated with the inward depolarizing current) ion channel protein-coding genes (Fig. 2c). The results further inspired us to characterize multiple parameters modulated during the process of
hiPSC-CMs maturation. Therefore, $\alpha$-actinin (Z-disk protein) was stained to visualize the siNC and siNRF2 hiPSC-CMs $(n>15$ cells per condition, three biological replicates) (Fig. 2d). Significant decreases in cell perimeter (siNRF2, $\quad 110.7 \pm 3.242 \mu \mathrm{m}$ vs. siNC, $142.5 \pm$ $6.676 \mu \mathrm{m} ; \quad P<0.0001)$, cell area (siNRF2, $834.5 \pm$ $66.34 \mu \mathrm{m}^{2}$ vs. siNC, $\left.1391 \pm 138 \mu \mathrm{m}^{2} ; \quad P<0.001\right)$, and sarcomere length (siNRF2, $1.663 \pm 0.018 \mu \mathrm{m}$ vs. siNC, $1.747 \pm 0.023 \mu \mathrm{m} ; P<0.01$ ) were observed in the siNRF2 hiPSC-CMs compared with the siNC hiPSC-CMs (Fig. $2 \mathrm{e}-\mathrm{g})$. The circularity index $\left.[4 \pi \text { area/(perimeter })^{2}\right]$ ("0" represents a theoretical minimum for perfect rodshaped cells, with " 1 " for perfectly round cells) increased from $0.77 \pm 0.013$ in siNC hiPSC-CMs to $0.82 \pm 0.01$ in the siNRF2 hiPSC-CMs, which indicated that the cells 


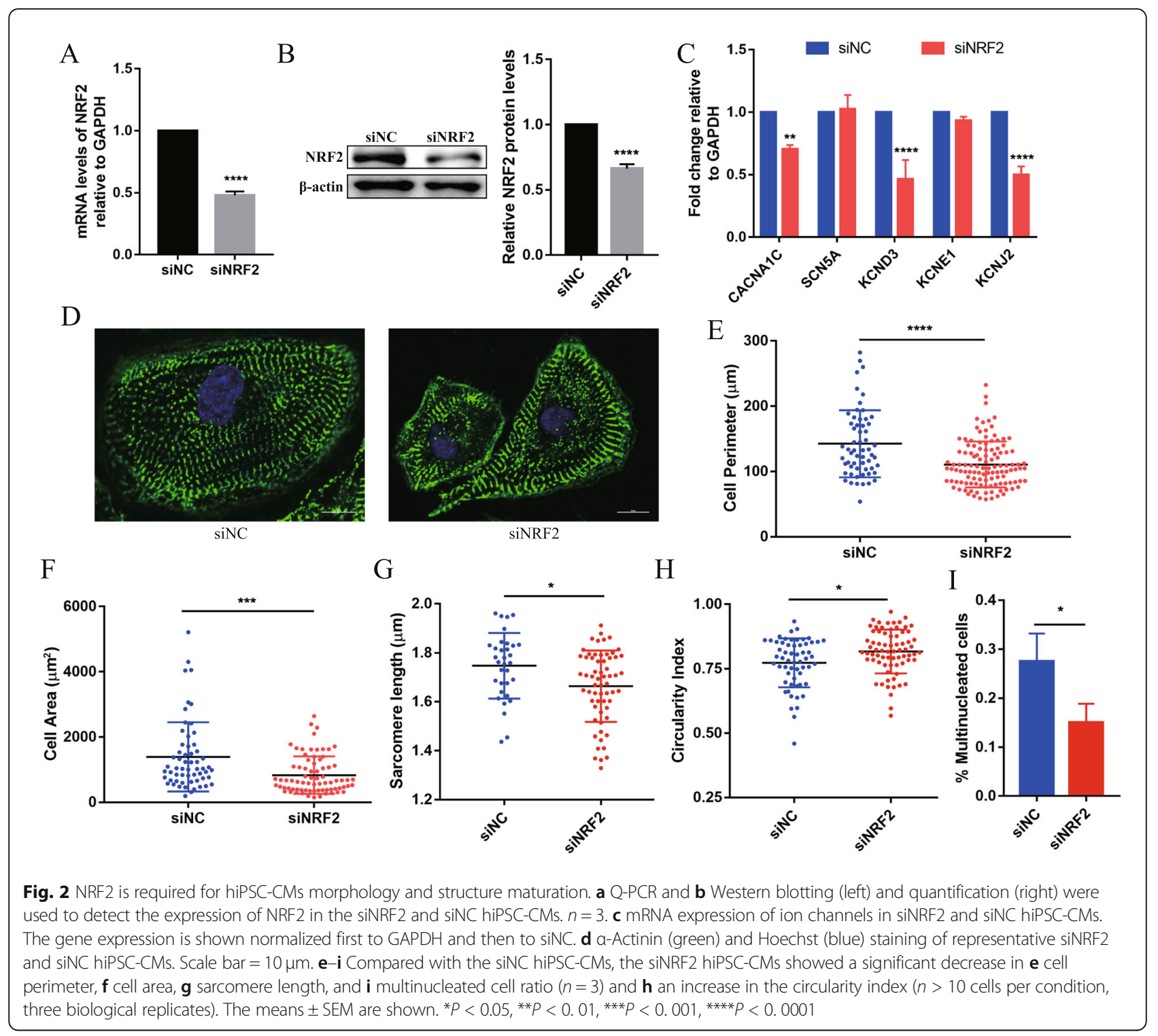

transformed into a round immature form (Fig. 2h). In addition, although human CMs do not proliferate, they are capable of DNA synthesis without nuclear division or nuclear division without cytokinesis, thereby increasing in ploidy $(8 \mathrm{~N})$ and size (hypertrophy) $[19,20]$. Hoechst staining was employed to analyze the number of nuclei, and the results showed that compared with the siNC cells, the proportion of multinuclear cells in siNRF2 hiPSC-CMs was significantly reduced (siNRF2, $0.1504 \pm 0.022$ vs. siNC, $0.2754 \pm 0.033 ; P<0.05$ ).

In addition, the calcium transient kinetics were assessed during contraction of hiPSC-CMs with Fluo-4 AM. These results showed that reduced peak amplitude, upstroke, and decay velocities were obtained in the siNRF2 hiPSC-CMs compared with the siNC hiPSCCMs (Fig. S2A-D), indicating that the calcium handling system was weakened. These results demonstrated that the morphologic and functional maturation of hiPSCCMs was arrested after NRF2 inhibition.

\section{Inhibition of NRF2 suppresses hiPSC-CM mitochondrial maturation}

To further analyze the effect of NRF2 on hiPSC-CM mitochondrial maturation, the morphology, number, and function of mitochondrial were evaluated after NRF2 inhibition. Research has shown that the abundance of mitochondria is increased with the maturity of the myocardium to meet higher energy demands [21]. Thus, we measured the abundance of mitochondrial DNA (mtDNA) by normalizing mtDNA to genomic nuclear DNA (gDNA). The mtDNA/gDNA ratio was significantly lower in the siNRF2 hiPSC-CMs than that in the 
siNC cells (siNRF2, $1.39 \pm 0.012$ vs. siNC, $1.45 \pm 0.013$; $P<0.05)$ (Fig. S3A). Accumulating evidence suggests that mitochondrial fusion plays a central role in the development of cardiomyocytes [22]. Therefore, we examined mitochondrial of live cells stained with MitoTracker Red by confocal laser scanning microscopy. As shown in Fig. S3B, the shape of mitochondria was transformed from an elongated to a punctiform phenotype, illustrating the reduction in mitochondrial fusion in the NRF2-inhibited cells compared with the siNC cells. The JC-1 fluorescent probe is used as an indicator of mitochondrial membrane potential $(\Delta \Psi \mathrm{m})$, which is also an important parameter used to assess the functional state of these organelles. The inhibition of NRF2 resulted in a reduction in mitochondrial membrane potential compared to that in the siNC cells (siNRF2, $0.557 \pm 0.029$ vs. siNC, $0.849 \pm 0.056 ; P<0.0001$ ) (Fig. S3C, D).

We also evaluated mitochondrial function in NRF2inhibited hiPSC-CMs by the XFe24 Cell Mito Stress Test (Seahorse Bioscience) and Substrate Oxidation Stress Test Kit (Fig. 3a, c). The results of oxygen consumption rate (OCR) were normalized based on the protein content. In the Mito Stress Test, the basal OCR was significantly lower in the siNRF2 hiPSC-CMs than in siNC hiPSC-CMs (Fig. 3a). The maximum respiration rate and spare respiratory capacity were also significantly lower in the siNRF2 hiPSC-CMs than in the siNC hiPSC-CMs (Fig. 3b). However, no significant difference in ATP production was observed between the siNRF2 hiPSC-CMs and siNC hiPSC-CMs (Fig. 3b). Similarly, the level of proton leakage, a sign of mitochondrial damage and a mechanism to regulate mitochondrial ATP production, did not significantly differ between the siNRF2 hiPSCCMs and siNC hiPSC-CMs (Fig. 3b). In the Substrate Oxidation Stress Test, Etomoxir, a specific inhibitor of carnitine palmitoyl transferase 1A (CPT1A), was used to specifically inhibit mitochondrial FAO. After Etomoxir treatment, the maximum respiration rate, spare respiratory capacity, ATP production, and proton leakage did not decrease in NRF2-inhibited hiPSC-CMs comparing with siNC hiPSC-CMs (Fig. 3d), indicating the fatty acid oxidation energy of siNRF2 hiPSC-CMs did not elevate. While the glucose consumption and lactic acid generation of siNRF2 hiPSC-CMs significantly increased (Fig. 3e, f). This result suggests that the knock-down of NRF2 led to the decrease of mitochondria respiration and increase of glycolysis.

In addition, we also tested the expression of genes related to glycolysis, oxidative phosphorylation, and fatty acid $\beta$-oxidation. Hexokinase (HK2), phosphofructokinase (PFK), and pyruvate kinase (PKM) used as surrogates for glycolysis rates were significantly increased in siNRF2 hiPSC-CMs compared with the control cells (Fig. 3g). In contrast with the high expression of these glycolysis genes, the expression of acetyl-CoA acyltransferase 2 (ACAA2) and carnitine palmitoyl transferase 1A (CPT1A), which encode key mitochondrial proteins involved in the $\beta$ oxidation of fatty acids, were dramatically reduced (Fig. 3h). In addition, the expression levels of mitochondrial pyruvate carrier 1 (MPC1), pyruvate dehydrogenase E1 subunit alpha 1 (PDHA1), citrate synthase (CS), isocitrate dehydrogenase (IDHA1), and oxoglutarate dehydrogenase $(\mathrm{OGDH})$, involved in aerobic oxidation regulation, were significantly reduced in siNRF2 hiPSC-CMs compared with the control cells (Fig. 3i). These data indicate that NRF2 is essential for the mitochondrial maturation of hiPSC-CMs.

\section{Activation of NRF2 enhances hiPSC-CMs maturation}

Under normal physiological conditions, NRF2 forms an E3 ubiquitin ligase complex consisting of cullin-3 (Cul3) with Kelch-like ECH-related protein 1 (KEAP1) outside the nucleus and is ubiquitinated and degraded. When dissociates from Keap1 and is translocated to the nucleus, NRF2 forms a heterodimer with the small Maf protein, binds to the antioxidant response element (ARE) in the promoter region of the target gene, and initiates transcription [23-25]. To further examine the effect of NRF2 on hiPSC-CMs maturation, we activated NRF2 by knocking down KEAP1 expression. After transfection of siKEAP1, the mRNA and protein expression levels of KEAP1 in hiPSC-CMs were significantly reduced (Fig. 4a, b), while the total protein expression levels of NRF2 and phosphorylated NRF2 (PS40-NRF2) were significantly increased (Fig. 4b), indicating that KEAP1 knock-down was effective and NRF2 was successfully activated. Then, the expression levels of the ion channel-related genes that activated by NRF2 in hiPSC-CMs were tested. These results showed that after NRF2 activation, the expression of genes encoding sodium (SCN5A), potassium (KCND3, KCNJ2 and KCNE1), and calcium channels (CACNA1C) was significantly increased, especially for CACNA1C, which was 6fold higher than that of siNC (Fig. 4c). In addition, immunocytochemical analyses with $\alpha$-actinin for Z-disk protein staining and Hoechst for nuclear counterstaining showed that NRF2 activation led to maturation changes in hiPSC-CMs. We observed a significant increase in cell perimeter (siKEAP1, $236.6 \pm 9.46 \mu \mathrm{m}$ vs. siNC, $131.6 \pm$ $8.48 \mu \mathrm{m}, P<0.0001$ ), cell area (siKEAP1, $3452 \pm 269 \mu \mathrm{m}^{2}$ vs. siNC, $\left.1392 \pm 138 \mu^{2}, P<0.0001\right)$, sarcomere length (siKEAP1, $1.9 \pm 0.015 \mu \mathrm{m}$ vs. siNC, $1.718 \pm 0.032 \mu \mathrm{m}, P<$ 0.0001 ), and proportion of cells with multiple nuclei (siKEAP1, $0.4064 \pm 0.039$ vs. siNC, $0.2202 \pm 0.044, P<$ $0.05)$ in NRF2-activated cells compared with siNC (Fig. 4d-h). Moreover, NRF2 activation resulted in a decreased circularity index (siKEAP1, $0.719 \pm 0.012$ vs. siNC, $0.777 \pm 0.013 P=0.0017$ ) (Fig. 4i). These data indicated 


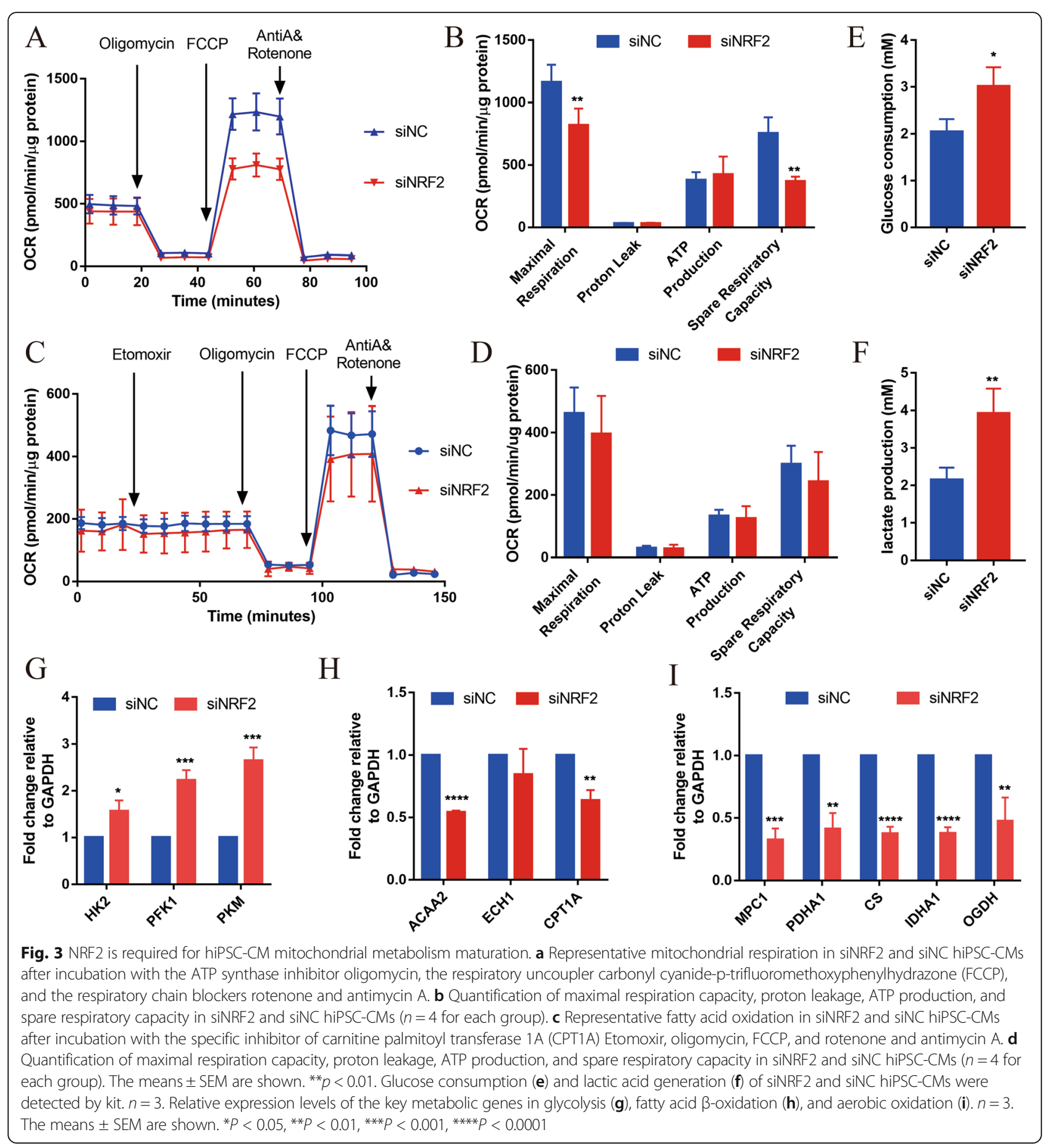

that NRF2 promoted the morphology maturation of hiPSC-CMs.

Cardiomyocytes in the embryonic period undergo a certain level of proliferation, and the proliferative ability is significantly weakened after maturation. EdU was employed to detect the cell proliferation level, and the results showed that the proliferation level of NRF2activated cells was significantly lower than that of siNC cells (Fig. 5a and Fig. S4A). Studies have reported that myosin heavy chain 6 (MyH6, fast-twitch) converts to myosin heavy chain 7 (MyH7; slow-twitch) during the process of myocardial maturation [26, 27]. We observed a significant decrease in $\mathrm{MyH} 6 / \mathrm{MyH} 7$ in the siKEAP1 hiPSC-CMs (siKEAP1, $1.032 \pm 0.004$ vs. siNC, $1.05 \pm$ $0.001, P<0.01)$ in NRF2-activated cells compared with control cells (Fig. 5b). Compared with siNC cells, the calcium transient kinetics of NRF2-activated hiPSC-CMs presented significant increases in peak calcium 


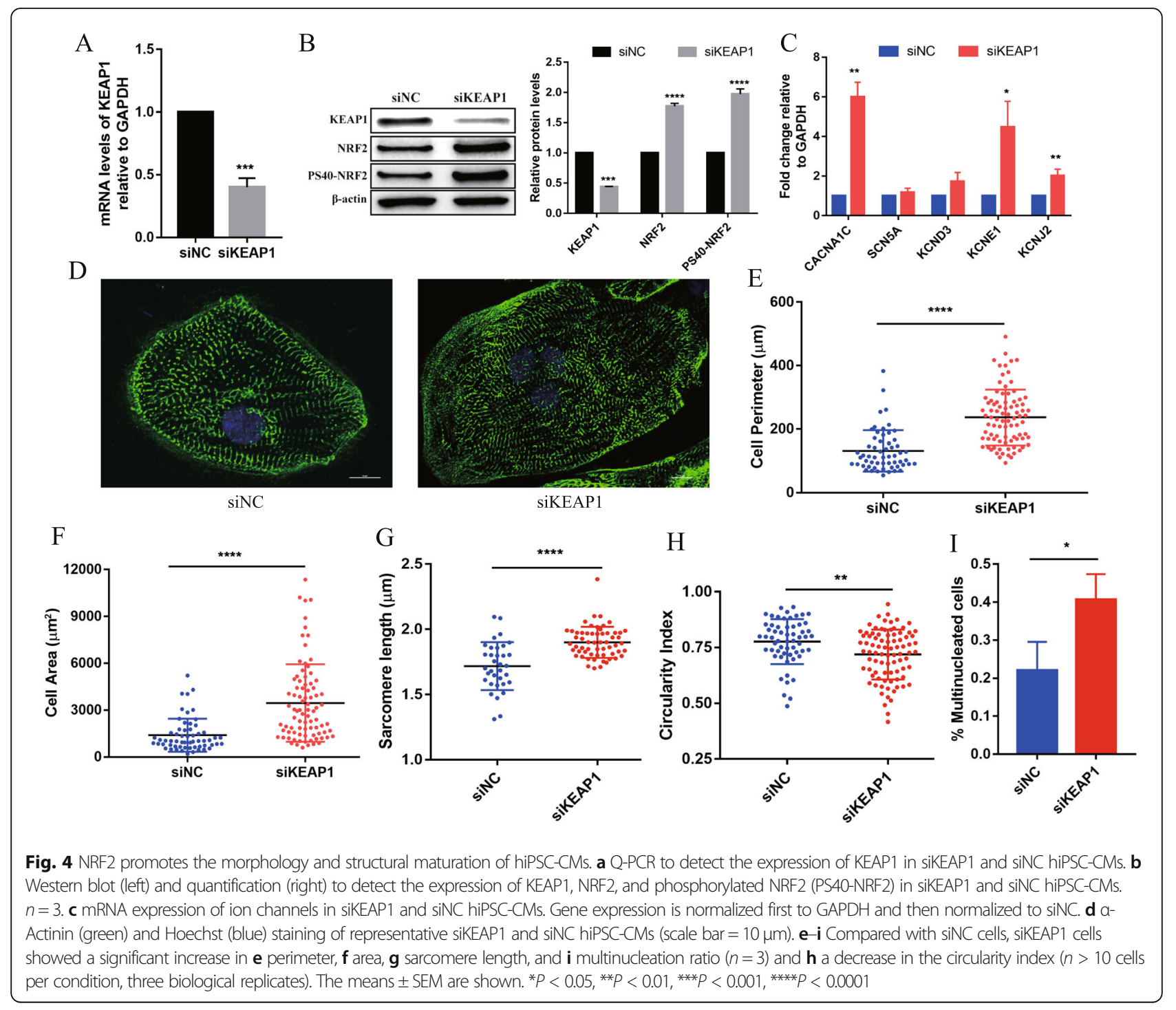

amplitude and faster $\mathrm{Ca}^{2+}$ transient upstroke velocities indicating calcium transient kinetics (Fig. 5c-e), which were consisted with elevated CACNA1C levels. These data are indicative of a more mature calcium transport system. However, the decay velocities were not significantly different (Fig. 5f). In summary, the function of hiPSC-CMs became more mature after activation of NRF2.

\section{Activation of NRF2 enhances hiPSC-CM mitochondrial maturation}

Studies have shown that NRF2 promotes mitochondrial biogenesis. Test results showed that the mtDNA copy number was significantly higher in the siKEAP1 hiPSCCMs than that in the siNC hiPSC-CMs (siKEAP1, $1.445 \pm$ 0.009 vs. siNC, $1.415 \pm 0.004, P<0.05$ ) (Fig. S5A). The MitoTracker Red stain showed that mitochondria changed from an elongated to a network phenotype, indicating that the degree of mitochondrial fusion was significantly increased in the NRF2-activated cells compared to that in the control cells (Fig. S5B). The JC-1 test results showed that NRF2 activation resulted in an increase in mitochondrial membrane potential compared to that in siNC cells (siKEAP1, $1.234 \pm 0.062$ vs. siNC, $0.949 \pm 0.037, P<0.001$ ) (Fig. S5C, E). These data suggest that mitochondrial morphology and structure were more mature in NRF2activated hiPSC-CMs.

To further verify whether the function of mitochondria is enhanced after NRF2 activation, the Agilent Seahorse XF Cell Mito Stress Test Kit and Substrate Oxidation Stress Test Kit were employed to test the mitochondrial function of hiPSC-CMs after NRF2 activation. In the Mito Stress Test, the basal OCR was significantly increased in the siKEAP1 hiPSC-CMs than which in the siNC hiPSC-CMs (Fig. 6a), and the production of ATP was also significantly higher in the siKEAP1 hiPSC-CMs, 

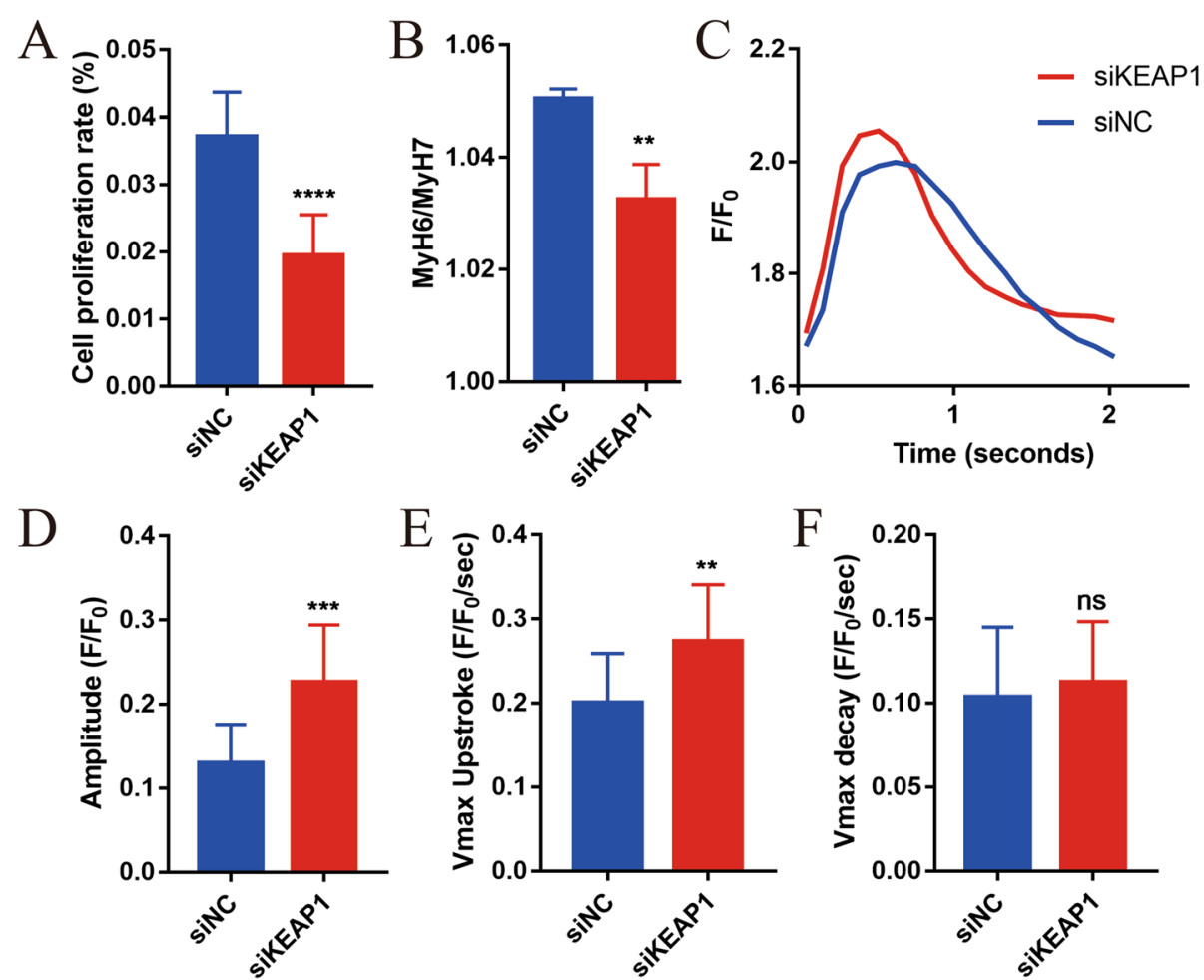

Fig. 5 NRF2 promotes function maturation of hiPSC-CMs. a Quantification of the percentage of sikEAP1 and siNC hiPSC-CMs proliferating, as measured by EdU (each biological replicate $n>5$, three biological replicates). $\mathbf{b}$ The proportion of MyH6 to MyH7 was decreased in the siKEAP1 hiPSC-CMs. $n=3$. $\mathbf{c}$, d HiPSC-CMs were analyzed by calcium transient kinetics with Fluo-4 AM ( $n>3$ cells per condition, three biological replicates): $\mathbf{c}$ representative calcium transient and $\mathbf{d}$ calcium transient amplitude (F/FO). e Maximum calcium transient upstroke velocities. $\mathbf{f}$ Maximum calcium transient decay velocities. The means \pm SEM are shown. ${ }^{* *} P<0.01,{ }^{* * *} P<0.001,{ }^{* * * *} P<0.0001$

which was consisted with the results determined by an ATP detection kit (Fig. 6b, S5D). In addition, as shown in Fig. 6b, the maximum respiration rate (siKEAP1, $1089 \pm 160.5 \mathrm{pmol} / \mathrm{min}$ vs. siNC, $689.2 \pm 69.45 \mathrm{pmol} / \mathrm{min}$, $P=0.0842$ ) and spare respiratory capacity (siKEAP1, $620.4 \pm 129.8 \mathrm{pmol} / \mathrm{min}$ vs. siNC, $389.1 \pm 33.77 \mathrm{pmol} /$ min, $P=0.1597)$ were higher in the siKEAP1 than that in the siNC hiPSC-CMs, but the differences were not statistically significant. And in the Substrate Oxidation Stress Test, the increased fatty acid oxidation energy of siKEAP1 hiPSC-CMs was shown by the decrease in oxygen consumption rate upon incubation with etomoxir (Fig. 6c). The maximum respiration rate, spare respiratory capacity, and the production of ATP were lower in the siKEAP1 than that in the siNC hiPSC-CMs (Fig. 6d). In addition, the glucose consumption and lactic acid generation was significantly decreased in the siKEAP1 hiPSC-CMs than which in the siNC hiPSC-CMs (Fig. 6e, f). Taken together, NRF2-activated hiPSC-CMs exhibited increasing mitochondrial respiratory and inhibited glycolysis compared with the siNC hiPSC-CMs.

It has been reported that NRF2 can inhibit fatty acid synthesis and promote fatty acid $\beta$-oxidation [14]. Our results showed that there was no difference in the expression of key glycolysis enzymes in the siKEAP1 and siNC cells (Fig. 6g), but the expression levels of genes that catalyze fatty acid $\beta$-oxidation, the tricarboxylic acid cycle (CS and IDHA1), and the mitochondrial respiratory complex subunits (complex II (succinate-coenzyme Q reductase, SDHB), complex III (coenzyme Qcytochrome c reductase, Cyt-C), complex IV (cytochrome c oxidase, COX5B), and complex V (ATP synthase, ATP5A)) were significantly increased in siKEAP1 (Fig. 6h-j).

\section{Bioinformatics analysis of the possible downstream target genes of NRF2}

To analyze the target genes that NRF2 directly regulates, the Cistrome Data Browser and GTRD databases were used to figure out the genes related to energy metabolism and myocardium maturation among the downstream genes targeted by NRF2. As shown in Tables 1 and 2, the results showed that NRF2 may directly target genes related to fatty acid $\beta$-oxidation metabolism (CPT1A and CPT2), mitochondrial respiratory (IDH, IDH2 complex I (NADHFS, NDUFA2, NDUFA5, NDUFA10, NDUFB2), complex II (SDHB), complex IV (COX6A1, COX5A), complex V (ATP5F1B, ATP5B, 


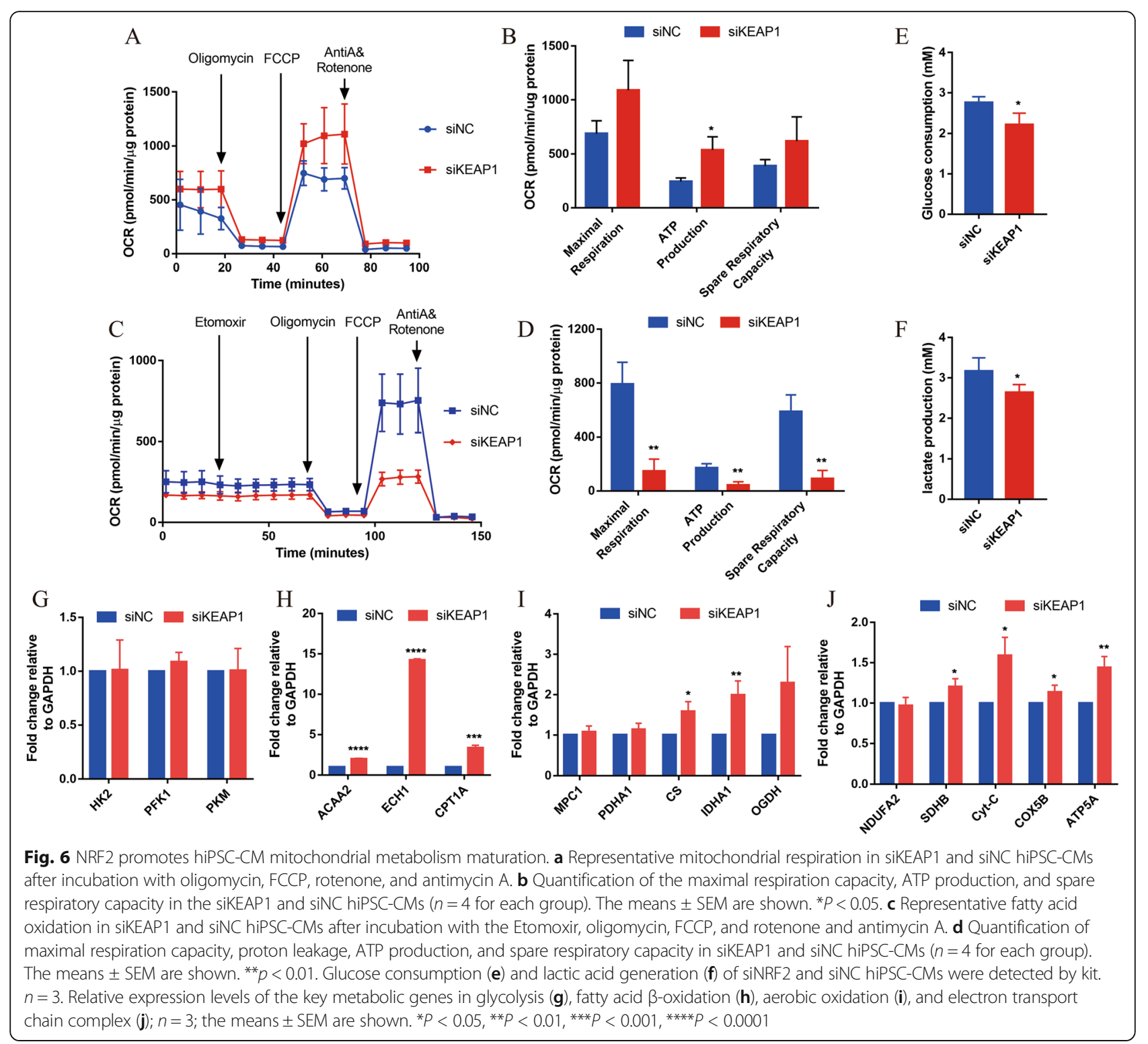

Table 1 Genes related to energy metabolism and myocardial maturation that NRF2 may bind to in the Cistrome Data Browser

\begin{tabular}{llll}
\hline Gene & Score & Biological source & Publication \\
\hline KCNC3 & 0.011 & GM06993, GM12872; lymphoblastoid; blood & Chorley et al., Nucleic Acids Res. 2012 \\
KCNA7 & 0.001 & GM06993, GM12872; lymphoblastoid; blood & Chorley et al., Nucleic Acids Res. 2012 \\
KCND3 & 0.997 & GM11992, GM12763; lymphoblastoid; blood & Chorley et al., Nucleic Acids Res. 2012 \\
CPT1A & 2.006 & HepG2; hepatocellular carcinoma & ENCODE Project Consortium et al., Nature 2012 \\
NDUFS4 & 0.936 & JHU-06; endothelial cell & Hogan et al., Elife \\
COX6A1 & 2.105 & LoVo; colon & Yan et al., Cell 2013 \\
ATP5F1B & 0.73 & GM07000, GM11882; lymphoblastoid; blood & Chorley et al., Nucleic Acids Res. 2012 \\
\hline
\end{tabular}


Table 2 Genes related to energy metabolism and myocardial maturation that may bind NRF2 as obtained from the GTRD database

\begin{tabular}{llll}
\hline ID & Gene symbol & Ensembl ID & SiteCount \\
\hline ENSG00000138413 & IDH1 & ENST00000446179 & 1 \\
ENSG00000182054 & IDH2 & ENST00000560061 & 1 \\
ENSG00000157184 & CPT2 & ENST00000468572 & 1 \\
ENSG00000131495 & NDUFA2 & ENST00000252102 & 1 \\
ENSG00000128609 & NDUFA5 & ENST00000466896 & 1 \\
ENSG00000130414 & NDUFA10 & ENST00000252711 & 2 \\
ENSG00000090266 & NDUFB2 & ENST00000247866 & 1 \\
ENSG00000073578 & SDHA & ENST00000264932 & 1 \\
ENSG00000178741 & COX5A & ENST00000562233 & 1 \\
ENSG00000110955 & ATP5B & ENST00000548647 & 1 \\
ENSG00000116459 & ATP5F1 & ENST00000464154 & 1 \\
ENSG00000078401 & EDN1 & ENST00000379375 & 1 \\
ENSG00000107147 & KCNT1 & ENST00000371757 & 1 \\
ENSG00000152049 & KCNE4 & ENST00000488477 & 1 \\
\hline
\end{tabular}

ATP5FA), potassium ion channel genes (KCNC3, KCNA7, KCND3, KCNT1, KCNE4), and cardiac hypertrophy (EDN1).

\section{Discussion}

hiPSC-CMs show spontaneous beating, but their metabolism and functional structure are most similar to those of immature cardiomyocytes in the embryonic period, which limits the further application of hiPSC-CMs in drug screening, disease modeling, and alternative treatment. Currently, most efforts to improve the maturation of hiPSC-CMs focused on biophysical, biochemical, and bioelectrical stimulation with genetic, chemical, and biomechanical approaches, and the study of energy substrates and metabolic pathways for stem cell differentiation of cardiomyocytes is limited [28-30]. It has been shown that the maturation of mitochondrial morphology and function is crucial for the development of hiPSCCMs $[31,32]$. Supplementing free fatty acids (FA) to the medium was a classic way to promote maturation of hiPSC-CMs, by which induced maturation of hiPSC$\mathrm{CMs}$ was even used as a positive control for HIF- $1 \alpha$ promoted maturation of hiPSC-CMs [8, 33]. A certain study reported that the expression level of NRF2 was significantly increased in hiPSC-CMs supplemented with FA, indicating the importance of NRF2 in the maturation of hiPSC-CMs [10].

As a transcription factor, NRF2 is related to the expression of more than 500 genes and engages enzymes that regulate mitochondrial respiration [34-36]. The significance of NRF2 in intermediary metabolism is seizing the interest of researchers. In various cellular contexts,
NRF2 control multiple mitochondrial functions, most notably the oxidative phosphorylation metabolism by binding to promoter regions of nuclear genes encoding subunits of the five respiratory complexes of the OXPHOS system, thus enhancing OXPHOS respiration and elevates intracellular ATP concentrations [9, 35]. The increased mitochondrial respiratory function is essential for the maturation of hiPSC-CMs. Consistently, after activation of NRF2, we found that the expression of mitochondrial respiratory subunits in hiPSC-CMs was significantly increased, accompanied by enhanced mitochondrial respiration and mitochondrial membrane potential. Out of expectation, the ATP production is not affected by the knock-down of NRF2, we assume that there is a compensatory mechanism involved in this process to meet the energy required for spontaneous beating, but further investigation is needed. NRF2 is also critical in the biogenesis of mitochondria, represented by increasing of the mitochondrial DNA (mtDNA) copy number, which coincides with our result [37, 38]. Mitsuishi et al. found that NRF2 status affects the genes encoding enzymes related with pentose phosphate pathway, which might be the reason why the number of multinucleated cells increased significantly after the activation of NRF2 we detected [39]. By analyzing and detecting the structure and function of cells and mitochondria in hiPSC-CMs, we preliminarily proved that NRF2 plays an important role in promoting the maturation of hiPSC-CMs. However, as only one single hiPSC cell line has been investigated in the present study, it remains to be elucidated whether NRF2 will play a similar role in other hiPSC cell lines of different origin. Further evaluation on more hiPSC cell lines from diverse source to conform the effect of NRF2 is currently on the schedule in our group.

On the other hand, in regenerative medicine and alternative therapy, transplanted hiPSC-CMs die quickly due to severe inflammation at the site of myocardial injury. ROS is considered to be a main cause of cell death. NRF2 inducers were found to exhibit a protective effect in cardiac remodeling. Cell culture models of cardiomyocytes have revealed the cytoprotective effect of NRF2 via reduction of ROS and inducing the production antioxidant and detoxification enzymes. Coincidently, the activation of NRF2 with resveratrol or curcumin showed a protective effect against inflammation and endothelial cell dysfunction $[15,40]$. Therefore, we speculated that enhanced NRF2 expression in hiPSC-CMs might also reduce the inflammatory response at the injured site. Bioinformatics analysis also revealed that NRF2 may regulate genes such as ion channels in addition to mitochondrial respiration. Accordingly, we hypothesize that NRF2 not only promotes cell maturation by changing mitochondrial respiration, but it may 
also combine multiple pathways to promote hiPSC-CMs maturation. Based on the above, the mechanism of NRF2 will be further screened and verified through ChIP-seq.

\section{Conclusions}

This study demonstrated that NRF2 is indispensable for the maturation of hiPSC-CMs, and the activation of NRF2 can be used as a key technical means to enhance the maturity of hiPSC-CMs. This improvement in understanding the mechanism of hiPSC-CMs maturity promotes potential applications of hiPSC-CMs in cardiac drug screening, disease modeling, and alternative treatments.

\begin{abstract}
Abbreviations
ACAA2: Acetyl-CoA acyltransferase 2; ATP5A, ATP5B, ATP5FA, ATP5F1B: ATP synthase (complex V); CACNA1C: Calcium ion channel; COX4, COX5A, COX5B, COX6A1: Cytochrome Coxidase subunits (complex IV); CPT1A and CPT2: Carnitine palmitoyl transferase; CS: Citrate synthase; CTnT: Cardiac troponin; CX43: Connexin43; Cyt-C: Coenzyme Q-cytochrome $\mathrm{c}$ reductase (complex III); ECH1: Enoyl-CoA hydratase 1; EDN1: Endothelin 1; FA: Fatty acid; FAO: Fatty acid oxidation; FCCP: Carbonyl cyanide-p-

trifluoromethoxyphenylhydrazone; GAPDH: Glyceraldehyde 3-phosphate dehydrogenase; GSK3- $\beta$ : Glycogen synthase kinase $3-\beta$; hiPSC-CMs: Human induced pluripotent stem cell-derived cardiomyocytes; HK2: Hexokinase; IDH, IDH2, IDHA1: Isocitrate dehydrogenase; KCNA7, KCNC3, KCND3, KCNE1, KCNE4, KCNJ2, KCNT1: Potassium ion channel; KEAP1: Kelch-like ECH-related protein 1; MPC1: Mitochondrial pyruvate carrier 1; MYH6, MYH7: Myosin heavy chain; MYL7: Myosin Light Chain; NDUFA1, NADHFS, NDUFA2, NDUFA5, NDUFA10, NDUFB2: NADH-coenzyme Q reductase (complex I); NRF2: Nuclear factor erythroid 2 p45-related factor 2; OCR: Oxygen consumption rate; OGDH: Oxoglutarate dehydrogenase; PDHA1: Pyruvate dehydrogenase E1 subunit alpha 1; PFK: Phosphofructokinase; PGM1: Pluripotency growth master 1; PKM: Pyruvate kinase muscle; QPCR: Quantitative real-time polymerase chain reaction; SCN5A: Sodium ion channel; SDHB: (Complex II) succinate-coenzyme Q reductase; SOX2: SRY-Box transcription factor 2; TNNT2: Troponin T2
\end{abstract}

\section{Supplementary Information}

Supplementary information accompanies this paper at https://doi.org/10. 1186/s13287-021-02264-2.

Additional file 2: Movie S1: Spontaneously beating of hiPSC-CMs. Additional file 1: Figure S1. Characterization of hiPSCs. (A) Cardiomyogenic differentiation protocol including hiPSC expansion, cardiomyocyte differentiation, purification, culture, and treatments. The different media and study factors used on different days of the timeline are indicated. (B) Immunostaining for SOX2 and Nanog in the hiPSCs. qPCR of OCT4 (C) and Nanog (D) during differentiation. The abscissa represents the number of days of differentiation. $n=3$; the means \pm SEM are shown. ${ }^{* * * * P}<0.0001$. Figure S2. siRNA knockdown of NRF2 decreased hiPSC-CMs functional maturation. hiPSC-CMs were analyzed by calcium transient kinetics evaluated with Fluo-4 AM. $n>3$ cells per condition, three biological replicates. (A) Representative calcium transient; (B) calcium transient amplitude (F/FO). (C) Maximum calcium transient upstroke velocities. (D) Maximum calcium transient decay velocities. The means \pm SEM are shown. ${ }^{* *} P<0.01,{ }^{* * *} P<0.0001$. Figure S3. siRNA knockdown of NRF2 decreased hiPSC-CMs mitochondrial maturation. (A) mtDNA copy numbers of siNRF2- and siNC-hiPSC-CMs were determined by qPCR; $n=3$. (B) Mitochondrial staining by MitoTracker Red (red, mitochondria; blue, nucleus); Scale bar $=10 \mu \mathrm{m}$. (C-D) Mitochondrial membrane potential $(m t \Delta \psi)$ of siNRF2 and siNC hiPSC-CMs are determined by JC-1 staining. (C) Quantitative analysis of the mitochondrial membrane potential $(m t \Delta \psi) ; n>4$ cells per condition, three biological replicates. (D) Representative $m t \Delta \psi$ in of siNRF2 and siNC hiPSC-CMs. Scale bar =
10 um. The means \pm SEM are shown. ${ }^{*} P<0.05,{ }^{* * * *} P<0.0001$. Figure S4. Cell proliferation of siKEAP1 and siNC hiPSC-CMs as analyzed by an BeyoClick ${ }^{\text {TM }}$ EdU cell proliferation kit with Alexa Fluor 488 and immunostaining of CTnT (red). Figure S5. NRF2 promotes the mitochondrial maturation of hiPSC-CMs. (A) mtDNA copy numbers of siKEAP1 and siNC hiPSC-CMs were determined by $\mathrm{PPCR}, \mathrm{n}=3$. (B) Mitochondrial staining by MitoTracker Red (red, mitochondria; blue, nucleus); Scale bar $=10 \mu \mathrm{m}$. (C, E) Mitochondrial membrane potential $(m t \Delta \psi)$ of the siKEAP1 and siNC hiPSC-CMs are determined by JC-1 staining. (C) Quantitative analysis of the mitochondrial membrane potential $(m t \Delta \psi) . n>8$ cells per condition, three biological replicates. (E) Representative mt $\Delta \psi$ in of the siKEAP1 and siNC hiPSC-CMs; Scale bar $=10 \mu \mathrm{m}$. (D) ATP content of the siKEAP1 and siNC hiPSC-CMs were determined using an ATP assay kit; $n=3$. The means \pm SEM are shown. ${ }^{*} P<0.05,{ }^{* * *} P<0.001$. Online Table 1. List of primers used for Q-PCR and mtDNA copy number.

\section{Acknowledgements}

Not applicable.

\section{Authors' contributions}

$\mathrm{XZ}$ contributed to the experimental conceptualization and design, performed all the experiments, analyzed and interpreted the data, and wrote the manuscript. $L Y, H X, Q Z$, and BT analyzed and interpreted the data and provided considerable manuscript review. JZ and JT contributed to the experimental design and revision of work. QY, LY, MX, and YZ contributed to the supervision of work and manuscript development. All authors read and approved the final manuscript.

\section{Funding}

This research was funded by the National Natural Science Foundation of China (grant number 81670270 and grant number 81700250 ) and the Science and Technology Research Program of Chongqing Municipal Education Commission (grant No. CYB19157).

\section{Availability of data and materials}

The datasets used and/or analyzed during the current study are available from the corresponding author on reasonable request.

\section{Declarations}

Ethics approval and consent to participate Not applicable.

\section{Consent for publication}

Not applicable.

\section{Competing interests}

The authors declare no competing interests.

\section{Author details}

${ }^{1}$ Department of Pediatric Research Institute, Ministry of Education Key Laboratory of Child Development and Disorders, National Clinical Research Center for Child Health and Disorders (Chongqing), China International Science and Technology Cooperation base of Child development and Critical Disorders, Children's Hospital of Chongqing Medical University, Chongqing, Box 136, No. 3 Zhongshan RD, Yuzhong district, Chongqing 400014, People's Republic of China. ${ }^{2}$ Chongqing Key Laboratory of Pediatrics, Chongqing, People's Republic of China. ${ }^{3}$ Department of Clinical Laboratory, Ministry of Education Key Laboratory of Child Development and Disorders, National Clinical Research Center for Child Health and Disorders (Chongqing), China International Science and Technology Cooperation base of Child development and Critical Disorders, Children's Hospital of Chongqing Medical University, Chongqing, People's Republic of China. ${ }^{4}$ Department of Cardiovascular (Internal Medicine), Ministry of Education Key Laboratory of Child Development and Disorders, National Clinical Research Center for Child Health and Disorders (Chongqing), China International Science and Technology Cooperation base of Child development and Critical Disorders, Children's Hospital of Chongqing Medical University, Chongqing, People's Republic of China. 
Received: 3 January 2021 Accepted: 2 March 2021

Published online: 24 March 2021

\section{References}

1. N Hellen, C Pinto Ricardo, K Vauchez, G Whiting, J Wheeler, S E Harding Proteomic analysis reveals temporal changes in protein expression in human induced pluripotent stem cell-derived cardiomyocytes in vitro. Stem Cells Dev. 2019;28(9):565-78. https://doi.org/10.1089/scd.2018.0210.

2. Breckwoldt K, Letuffe-Breniere D, Mannhardt I, Schulze T, Ulmer B, Werner T, Benzin A, Klampe B, Reinsch MC, Laufer S, Shibamiya A, Prondzynski M, Mearini G, Schade D, Fuchs S, Neuber C, Kramer E, Saleem U, Schulze ML, Rodriguez ML, Eschenhagen T, Hansen A. Differentiation of cardiomyocytes and generation of human engineered heart tissue. Nat Protoc. 2017;12(6): 1177-97. https://doi.org/10.1038/nprot.2017.033.

3. Zhou Y, Wang L, Liu Z, Alimohamadi S, Yin C, Liu J, Qian L. Comparative gene expression analyses reveal distinct molecular signatures between differentially reprogrammed cardiomyocytes. Cell Rep. 2017;20(13):3014-24. https://doi.org/10.1016/j.celrep.2017.09.005.

4. Karakikes I, Ameen M, Termglinchan V, Wu JC. Human induced pluripotent stem cell-derived cardiomyocytes: insights into molecular, cellular, and functional phenotypes. Circ Res. 2015;117(1):80-8. https://doi.org/10.1161/ CIRCRESAHA.117.305365.

5. Denning C, Borgdorff V, Crutchley J, Firth KS, George V, Kalra S, Kondrashov A, Hoang MD, Mosqueira D, Patel A, Prodanov L, Rajamohan D, Skarnes WC, Smith JG, Young LE. Cardiomyocytes from human pluripotent stem cells: from laboratory curiosity to industrial biomedical platform. Biochim Biophys Acta. 2016;1863(7):1728-48. https://doi.org/10.1016/j.bbamcr.2015.10.014

6. Nose N, Werner RA, Ueda Y, Gunther K, Lapa C, Javadi MS, Fukushima K Edenhofer F, Higuchi T. Metabolic substrate shift in human induced pluripotent stem cells during cardiac differentiation: functional assessment using in vitro radionuclide uptake assay. Int J Cardiol. 2018;269:229-34 https://doi.org/10.1016/j.ijcard.2018.06.089

7. Yoshida S, Tsutsumi S, Muhlebach G, Sourbier C, Lee MJ, Lee S, Vartholomaiou E, Tatokoro M, Beebe K, Miyajima N, Mohney RP, Chen Y, Hasumi H, Xu W, Fukushima H, Nakamura K, Koga F, Kihara K, Trepel J, Picard D, Neckers L. Molecular chaperone TRAP1 regulates a metabolic switch between mitochondrial respiration and aerobic glycolysis. Proc Nat Acad Sci U S A. 2013;110(17):E1604-12. https://doi.org/10.1073/pnas.122 0659110.

8. Hu D, Linders A, Yamak A, Correia C, Kijlstra JD, Garakani A, Xiao L, Milan DJ, van der Meer P, Serra M, Alves PM, Domian IJ. Metabolic maturation of human pluripotent stem cell-derived cardiomyocytes by inhibition of HIF1alpha and LDHA. Cell Biol. 2018;123:1066-79.

9. Holmström KM, Kostov RV, Dinkova-Kostova AT. The multifaceted role of NRF2 in mitochondrial function. Curr Opin Toxicol. 2016;1:80-91. https://doi. org/10.1016/j.cotox.2016.10.002.

10. Ramachandra CJA, Mehta A, Wong P, Ja K, Fritsche-Danielson R, Bhat RV, Hausenloy DJ, Kovalik JP, Shim W. Fatty acid metabolism driven mitochondrial bioenergetics promotes advanced developmental phenotypes in human induced pluripotent stem cell derived cardiomyocytes. Int J Cardiol. 2018;272:288-97. https://doi.org/10.1016/j.jica rd.2018.08.069.

11. Holmstrom KM, Baird L, Zhang Y, Hargreaves I, Chalasani A, Land JM, Stanyer L, Yamamoto M, Dinkova-Kostova AT, Abramov AY. NRF2 impacts cellular bioenergetics by controlling substrate availability for mitochondrial respiration. Biol Open. 2013;2(8):761-70. https://doi.org/10.1242/bio.20134 853.

12. A Abdullah, N R Kitteringham, R E Jenkins, C Goldring, L Higgins, M Yamamoto, J Hayes, B K Park. Analysis of the role of NRF2 in the expression of liver proteins in mice using two-dimensional gel-based proteomics. Pharmacol Rep. 2012;64(3):680-97. https://doi.org/10.1016/s1734-1140(12 )70863-0.

13. A S Agyeman, R Chaerkady, P G Shaw, N E Davidson, K Visvanathan, A Pandey, T W Kensler. Transcriptomic and proteomic profiling of KEAP1 disrupted and sulforaphane-treated human breast epithelial cells reveals common expression profiles. Breast Cancer Res Treat. 2012;132(1):175-87. https://doi.org/10.1007/s10549-011-1536-9.

14. Ludtmann MH, Angelova PR, Zhang Y, Abramov AY, Dinkova-Kostova AT. NRF2 affects the efficiency of mitochondrial fatty acid oxidation. Biochem J. 2014;457(3):415-24. https://doi.org/10.1042/BJ20130863.
15. Chen QM, Maltagliati AJ. NRF2 at the heart of oxidative stress and cardiac protection. Physiol Genomics. 2018;50(2):77-97. https://doi.org/10.1152/ physiolgenomics.00041.2017.

16. Tohyama S, Hattori F, Sano M, Hishiki T, Nagahata Y, Matsuura T, Hashimoto H, Suzuki T, Yamashita H, Satoh Y, Egashira T, Seki T, Muraoka N, Yamakawa H, Ohgino Y, Tanaka T, Yoichi M, Yuasa S, Murata M, Suematsu M, Fukuda K. Distinct metabolic flow enables large-scale purification of mouse and human pluripotent stem cell-derived cardiomyocytes. Cell Stem Cell. 2013; 12(1):127-37. https://doi.org/10.1016/j.stem.2012.09.013.

17. A Sharma, G Li, K Rajarajan, R Hamaguchi. Derivation of highly purified cardiomyocytes from human induced pluripotent stem cells using small molecule-modulated differentiation and subsequent glucose starvation. J Vis Exp. 2015:2015(97):52628. https://doi.org/10.3791/52628.

18. Lian X, Zhang J, Azarin SM, Zhu K, Hazeltine LB, Bao X, Hsiao C, Kamp TJ, Palecek SP. Directed cardiomyocyte differentiation from human pluripotent stem cells by modulating Wnt/beta-catenin signaling under fully defined conditions. Nat Protoc. 2013:8(1):162-75. https://doi.org/10.1038/nprot.2 012.150.

19. Ahuja P, Sdek P, MacLellan WR. Cardiac myocyte cell cycle control in development, disease, and regeneration. Physiol Rev. 2007;87(2):521-44. https://doi.org/10.1152/physrev.00032.2006.

20. G Olivetti, E Cigola, R Maestri, D Corradi, C Lagrasta, S R Gambert, P Anversa. Aging, cardiac hypertrophy and ischemic cardiomyopathy do not affect the proportion of mononucleated and multinucleated myocytes in the human heart. J Mol Cell Cardiol. 1996;28(7):1463-77. https://doi.org/10.1006/jmcc.1 996.0137.

21. B M Ulmer, T Eschenhagen. Human pluripotent stem cell-derived cardiomyocytes for studying energy metabolism. Biochim Biophys Acta Mol Cell Res. 2020;1867(3):118471. https://doi.org/10.1016/j.bbamcr.2019.04.001.

22. Green DR, Kroemer G. Mitochondrial fusion directs cardiomyocyte differentiation via calcineurin and notch signaling. Science. 2004;305(5684): 626-9. https://doi.org/10.1126/science.1099320.

23. Kensler TW, Wakabayashi N, Biswal S. Cell survival responses to environmental stresses via the Keap1-NRF2-ARE pathway. Annu Rev Pharmacol Toxicol. 2007;47(1):89-116. https://doi.org/10.1146/annurev.pha rmtox.46.120604.141046.

24. Zhang DD, Lo SC, Cross JV, Templeton DJ, Hannink M. Keap1 is a redoxregulated substrate adaptor protein for a Cul3-dependent ubiquitin ligase complex. Mol Cell Biol. 2004;24(24):10941-53. https://doi.org/10.1128/ MCB.24.24.10941-10953.2004

25. Itoh K, Wakabayashi N, Katoh Y, Ishii T, Igarashi K, Engel JD, Yamamoto M Keap1 represses nuclear activation of antioxidant responsive elements by NRF2 through binding to the amino-terminal Neh2 domain. Genes Dev. 1999;13(1):76-86. https://doi.org/10.1101/gad.13.1.76.

26. Xu XQ, Soo SY, Sun W, Zweigerdt R. Global expression profile of highly enriched cardiomyocytes derived from human embryonic stem cells. Stem Cells. 2009;27(9):2163-74. https://doi.org/10.1002/stem.166.

27. V MAHDAVI, A M LOMPRE, A P Chambers, B NADAL-GINARD. Cardiac myosin heavy chain isozymic transitions during development and under pathological conditions are regulated at the level of mRNA availability. Eur Heart J. 1984; 5(Suppl F):181-91. https://doi.org/10.1093/eurheartj/5.suppl_f.181.

28. Yang X, Pabon L, Murry CE. Engineering adolescence: maturation of human pluripotent stem cell-derived cardiomyocytes. Circ Res. 2014;114(3):511-23. https://doi.org/10.1161/CIRCRESAHA.114.300558.

29. Ronaldson-Bouchard K, Ma SP, Yeager K, Chen T, Song L, Sirabella D, Morikawa K, Teles D, Yazawa M, Vunjak-Novakovic G. Advanced maturation of human cardiac tissue grown from pluripotent stem cells. Nature. 2018; 556(7700):239-43. https://doi.org/10.1038/s41586-018-0016-3.

30. Correia C, Koshkin A, Duarte P, Hu D, Carido M, Sebastiao MJ, Gomes-Alves P, Elliott DA, Domian IJ, Teixeira AP, Alves PM, Serra M. 3D aggregate culture improves metabolic maturation of human pluripotent stem cell derived cardiomyocytes. Biotechnol Bioeng. 2018;115(3):630-44. https://doi.org/10.1 002/bit.26504

31. Gaspar JA, Doss MX, Hengstler JG, Cadenas C, Hescheler J, Sachinidis A Unique metabolic features of stem cells, cardiomyocytes, and their progenitors. Circ Res. 2014;114(8):1346-60. https://doi.org/10.1161/CIRCRESA HA.113.302021.

32. Mills RJ, Titmarsh DM, Koenig X, Parker BL, Ryall JG, Quaife-Ryan GA, Voges HK, Hodson MP, Ferguson C, Drowley L, Plowright AT, Needham EJ, Wang QD, Gregorevic P, Xin M, Thomas WG, Parton RG, Nielsen LK, Launikonis BS, James DE, Elliott DA, Porrello ER, Hudson JE. Functional screening in human 
cardiac organoids reveals a metabolic mechanism for cardiomyocyte cell cycle arrest. Proc Natl Acad Sci U S A. 2017;114(40):E8372-81. https://doi. org/10.1073/pnas.1707316114.

33. Yang $X$, Rodriquez ML, Leonard A, Sun L, Fischer KA, Wang Y, Ritterhoff J, Zhao L, Kolwicz SC Jr, Pabon L, Reinecke H, Sniadecki NJ, Tian R, RuoholaBaker $\mathrm{H}, \mathrm{Xu} \mathrm{H}$, Murry CE. Fatty acids enhance the maturation of cardiomyocytes derived from human pluripotent stem cells. Stem Cell Rep. 2019;13:657-68.

34. Mitsuishi Y, Taguchi K, Kawatani Y, Shibata T, Nukiwa T, Aburatani H, Yamamoto M, Motohashi H. NRF2 redirects glucose and glutamine into anabolic pathways in metabolic reprogramming. Cancer Cell. 2012;22(1):6679. https://doi.org/10.1016/j.ccr.2012.05.016.

35. Dinkova-Kostova AT, Abramov AY. The emerging role of NRF2 in mitochondrial function. Free Radic Biol Med. 2015;88(Pt B):179-88. https:// doi.org/10.1016/j.freeradbiomed.2015.04.036

36. Malhotra D, Portales-Casamar E, Singh A, Srivastava S, Arenillas D, Happel C, Shyr C, Wakabayashi N, Kensler TW, Wasserman WW, Biswal S. Global mapping of binding sites for NRF2 identifies novel targets in cell survival response through ChIP-Seq profiling and network analysis. Nucleic Acids Res. 2010;38(17):5718-34. https://doi.org/10.1093/nar/gkq212.

37. I-g R, Kwak M-K. Regulatory crosstalk between the oxidative stress-related transcription factor Nfe2l2/NRF2 and mitochondria. Toxicol Appl Pharmacol. 2018;359:24-33.

38. Gureev AP, Shaforostova EA, Popov VN. Regulation of mitochondrial biogenesis as a way for active longevity: interaction between the NRF2 and PGC-1alpha signaling pathways. Front Genet. 2019;10:435. https://doi.org/1 0.3389/fgene.2019.00435

39. Mitsuishi Y, Motohashi H, Yamamoto M. The Keap1-NRF2 system in cancers: stress response and anabolic metabolism. Front Oncol. 2012;2:200.

40. L Cheng, Z Jin, R Zhao, K Ren, C Deng, S Yu. Resveratrol attenuates inflammation and oxidative stress induced by myocardial ischemiareperfusion injury: role of NRF2/ARE pathway. Int J Clin Exp Med. 2015;8(7): 10420-8. eCollection 2015

\section{Publisher's Note}

Springer Nature remains neutral with regard to jurisdictional claims in published maps and institutional affiliations.

Ready to submit your research? Choose BMC and benefit from:

- fast, convenient online submission

- thorough peer review by experienced researchers in your field

- rapid publication on acceptance

- support for research data, including large and complex data types

- gold Open Access which fosters wider collaboration and increased citations

- maximum visibility for your research: over $100 \mathrm{M}$ website views per year

At $\mathrm{BMC}$, research is always in progress.

Learn more biomedcentral.com/submissions 